\title{
Efecto de un Plan de Mejora de Calidad y Seguridad Clínica en la incidencia de infección de sitio quirúrgico en apendicectomía. Estudio cuasi-experimental
}

\author{
Effect of a Plan for Quality Improvement and Clinical Safety in the incidence of surgical \\ site infections in appendectomy. A quasi-experimental study
}

\author{
Juan A. del-Moral-Luque ${ }^{1,2}$, Tomás Sánchez-Santana 3 , Pablo Gil-Yonte ${ }^{4}$, José M. Fernández-Cebrián", \\ Ana I. Hijas-Gómez ${ }^{5}$ y Gil Rodríguez-Caravaca ${ }^{1,3 *}$ \\ ${ }^{1}$ Unidad de Medicina Preventiva, Hospital Universitario Fundación Alcorcón; ${ }^{2}$ Programa Interuniversitario de Doctorado; ${ }^{3}$ Departamento de Medicina \\ Preventiva y Salud Pública, Universidad Rey Juan Carlos; ${ }^{4}$ Servicio de Cirugía General, Hospital Universitario Fundación Alcorcón; ${ }^{5}$ Agencia de \\ Evaluación de Tecnologías Sanitarias, Instituto de Salud Carlos III. Madrid, España
}

\section{Resumen}

Objetivo: Las infecciones de sitio quirúrgico se pueden evitar y los programas de control basados en paquetes de medidas preventivas son eficaces para reducir su incidencia. El objetivo de este estudio fue evaluar el efecto de un Plan de Mejora de Calidad y Seguridad Clínica del paciente intervenido de apendicectomía en la incidencia de infección del sitio quirúrgico. Método: Se realizó un estudio cuasi-experimental con análisis antes y después de la introducción de un Plan de Calidad y Seguridad Clínica. Se incluyeron pacientes intervenidos de apendicectomía. Se estudió la incidencia de infección del sitio quirúrgico durante los 30 días posteriores a la cirugía (periodo máximo de incubación de infección quirúrgica). Se evaluó el efecto de la intervención con la odds ratio (OR) ajustada con un modelo de regresión logística. Resultados: Se incluyeron 606 pacientes, 267 en el periodo 2009-2010 (antes del plan) y 339 durante 2012-2013 (después del plan). La incidencia de infección del sitio quirúrgico descendió después del plan del 6 al 5.6\% (OR: 0.72; intervalo de confianza del 95\%: 0.33-1.56; $p=0.839$ ). Hubo mayor cumplimiento de la profilaxis antibiótica, de la preparación prequirúrgica y de la adherencia a la higiene de manos tras la introducción de las medidas. Conclusiones: Aunque la reducción de la incidencia de infección del sitio quirúrgico no presentó diferencias estadísticamente significativas tras las medidas adoptadas, se ha conseguido mejorar la administración de la profilaxis antibiótica, la adherencia a la higiene de manos y la preparación prequirúrgica.

PALABRAS CLAVE: Apendicectomía. Infección de sitio quirúrgico. Paquete de medidas. Profilaxis antibiótica. Preparación prequirúrgica. Higiene de manos.

\begin{abstract}
Objective: Surgical site infections can be prevented. Control programs based on care bundle have proven to be effective in reducing its incidence. The objective of this study was to assess the effectiveness of a Plan for Quality Improvement and Clinical Safety in preventing the incidence of surgical site infection in patients undergoing appendectomy. Method: $A$ quasi-experimental study was designed for analysis before and after the introduction of a Plan for Quality and Clinical Safety. Patients undergoing appendectomy were included. The incidence of surgical site infection was studied within 30 days from the time of surgery (maximum incubation period of surgical site infection). The effectiveness of the intervention was evaluated using the odds ratio (OR) adjusted with a logistic regression model. Results: A total of 606 patients were included, of which 267 were operated in the period 2009-2010 (before the plan) and 339 in 2012-2013 (after the plan). The incidence of surgical site infection
\end{abstract}


decreased after the plan from 6 to 5.6\% (OR: 0.72; 95\% confidence interval: 0.33-1.56; $p=0.839$ ). There was greater compliance of antibiotic prophylaxis, preoperative preparation and adherence to hand hygiene after the introduction of the measures. Conclusions: Although the reduction in the incidence of surgical site infection after the measures adopted did not show statistical significant differences, important progress has been made in the compliance of antibiotic prophylaxis, adherence to hand hygiene and in the preoperative preparation.

KEY WORDS: Appendectomy. Surgical wound infection. Patient care bundle. Antibiotic prophylaxis. Preoperative preparation. Hand hygiene.

\section{Introducción}

Las infecciones nosocomiales son la complicación más frecuente durante el ingreso hospitalario de un paciente. Se estima que el $5 \%$ de los pacientes ingresados en un hospital adquieren una infección nosocomial $^{1}$, y que podrían prevenirse un tercio o más de ellas mediante distintas estrategias de vigilancia y control$^{2}$. La prevención de estas infecciones es más barata que su tratamiento posterior ${ }^{3}$.

La infección del sitio quirúrgico (ISQ), según los criterios de los Centers for Disease Control and Prevention de los EE.UU., es aquella infección relacionada con el procedimiento quirúrgico que se produce en la incisión quirúrgica o su vecindad, durante los 30 primeros días del posoperatorio (90 días en caso de colocación de implantes). La ISQ es la principal causa de infección nosocomial en los pacientes quirúrgicos ${ }^{4}$.

Actualmente, en España, la infección quirúrgica es la primera causa de infección nosocomial en los pacientes ingresados, seguida de las urinarias y de las respiratorias, con un $20 \%{ }^{5}$. La cirugía apendicular es, junto con la de colon, la de mayor riesgo de infección en el área abdominal ${ }^{6}$.

La incidencia de ISQ en apendicectomías varía entre el 2 y el $15 \%$,7, Los pacientes con ISQ tienen un $60 \%$ más de probabilidad de ingresar en una unidad de cuidados intensivos, cinco veces más de reingresar en el hospital y el doble de probabilidades de fallecer ${ }^{9}$. La presencia de ISQ aumenta, por tanto, el riesgo y la gravedad del paciente.

Para reducir la incidencia de la infección quirúrgica podemos actuar en tres momentos: en la fase prequirúrgica mediante la profilaxis antibiótica y la higiene preoperatoria, durante la cirugía con el cuidado de los tejidos y la optimización de la técnica quirúrgica, y después de la operación a través de una vigilancia epidemiológica mantenida durante el periodo máximo de incubación ${ }^{10,11}$.
Tabla 1. Procedimientos quirúrgicos estudiados (códigos de la Clasificación Internacional de Enfermedades, 9. a revisión, modificación clínica)

\begin{tabular}{ll}
\hline Código & Procedimiento quirúrgico \\
\hline 47.01 & Apendicectomía laparoscópica \\
47.09 & Otra apendicectomía \\
47.2 & Drenaje de absceso apendicular \\
47.91 & Apendicostomía \\
47.92 & Cierre de fístula apendicular \\
47.99 & Otro procedimiento quirúrgico en el \\
& apéndice \\
\hline
\end{tabular}

Las tasas de incidencia de ISQ son un indicador de calidad de la práctica quirúrgica, y los programas de control basados en paquetes de medidas preventivas (care bundle) son eficaces y costo-efectivos ${ }^{12}$. Conociendo la evidencia de dichas medidas, en nuestro hospital se implantó, durante el año 2011, un Plan de Mejora de Calidad y Seguridad Clínica del paciente intervenido de apendicectomía. El objetivo de este estudio fue evaluar el efecto de dicho plan en la incidencia de ISQ.

\section{Método}

Se realizó un estudio cuasi-experimental con análisis antes y después de la introducción de un Plan de Mejora de Calidad y Seguridad Clínica en el Hospital Universitario Fundación Alcorcón. El estudio incluyó los pacientes intervenidos de apendicectomía en el servicio de cirugía general y del aparato digestivo durante los periodos 2009-2010 (antes del plan) y 2012-2013 (después del plan). Los pacientes fueron seleccionados mediante inclusión consecutiva. En la tabla 1 se detallan los distintos procedimientos quirúrgicos de intervención del apéndice que se incluyeron en este estudio. 
Tabla 2. Clasificación de las infecciones del sitio quirúrgico según los criterios de los Centers for Disease Control and Prevention

\begin{tabular}{|c|c|}
\hline Tipo de infección & Criterios \\
\hline Infección superficial & $\begin{array}{l}\text { Aparición en los } 30 \text { días posteriores a la cirugía. Afecta solo a la piel o el tejido celular subcutáneo de la zona } \\
\text { de incisión y cumple al menos uno de los siguientes criterios: } \\
\text { - Drenaje purulento } \\
\text { - Cultivo positivo de exudado de la herida } \\
\text { - Diagnóstico médico de infección superficial } \\
\text { - El cirujano abre deliberadamente la incisión y el cultivo de esta es positivo o no existe cultivo, y además } \\
\quad \text { hay un síntoma o signo clínico de los siguientes: dolor, inflamación local, rubor o calor }\end{array}$ \\
\hline Infección profunda & $\begin{array}{l}\text { Aparición en los } 30 \text { días posteriores a la cirugía. Afecta a los tejidos profundos de la incisión (fascia y paredes } \\
\text { musculares) y cumple al menos uno de los siguientes criterios: } \\
\text { - Drenaje purulento de la zona profunda de la incisión } \\
\text { - Dehiscencia espontánea o apertura de la herida por el cirujano y cultivo positivo o no había cultivo, y el } \\
\text { paciente tiene al menos uno de los siguientes signos o síntomas: fiebre, dolor local o hipersensibilidad al } \\
\text { tacto o la presión } \\
\text { - Diagnóstico médico de infección profunda } \\
\text { - Absceso diagnosticado por examen directo de la incisión, en una reintervención o por estudio } \\
\text { histopatológico o radiológico }\end{array}$ \\
\hline $\begin{array}{l}\text { Infección de } \\
\text { órgano-espacio }\end{array}$ & $\begin{array}{l}\text { Aparición en los } 30 \text { días posteriores a la cirugía. Afecta a cualquier parte de la anatomía distinta de la incisión } \\
\text { y cumple uno de los siguientes criterios: } \\
\text { - Secreción purulenta en un drenaje colocado en un órgano o espacio } \\
\text { - Cultivo positivo de muestras de fluidos o de tejidos procedentes de órganos o espacios } \\
\text { - Absceso u otra evidencia de infección obtenida por examen directo de la incisión, reintervención o estudio } \\
\text { histopatológico o radiológico, que afecte a órgano o espacio } \\
\text { - Diagnóstico médico de infección quirúrgica de órgano o espacio }\end{array}$ \\
\hline
\end{tabular}

El tamaño muestral se calculó según un nivel de confianza del $80 \%$, una potencia del $80 \%$, una incidencia de infección del $7 \%$ en el grupo sin intervención y del $3 \%$ en el grupo con intervención, y unas pérdidas durante el seguimiento del $5 \%$. Se estimó necesaria una muestra de 534 pacientes. El estudio fue aprobado por del Comité de Ética e Investigación Clínica del hospital.

Se estudió la evolución de los pacientes desde el momento de la cirugía hasta el final del periodo de incubación máximo de 30 días. Se utilizaron los criterios de los CDC para el diagnóstico de ISQ (Tabla 2). El riesgo de infección quirúrgica se estimó con el índice del National Nosocomial Infections Surveillance (NNIS), que utiliza el valor del riesgo anestésico de la American Society of Anesthesiologists (ASA), el grado de contaminación quirúrgica y la duración de la cirugía mayor del percentil 75. La evolución clínica de cada paciente se estudió revisando su historia clínica durante el ingreso para ver la evolución diaria de la herida quirúrgica, y los cultivos microbiológicos si se hubieran solicitado. En el caso de los pacientes ingresados, la ISQ fue evaluada por un médico especialista en medicina preventiva y un cirujano. Tras el alta de los pacientes se realizó un seguimiento activo revisando sus visitas periódicas a las consultas externas de cirugía general y del aparato digestivo, urgencias y centros de atención primaria, mediante la aplicación Horus ${ }^{\circledR}$ de acceso a la historia clínica.

Las variables incluidas en el estudio fueron la edad, la obesidad (índice de masa corporal $\geq 30$ ), la diabetes mellitus, la insuficiencia renal, la neoplasia, la enfermedad pulmonar obstructiva crónica, la neutropenia, la cirrosis hepática, la desnutrición, la duración de la cirugía, la transfusión sanguínea, el drenaje posquirúrgico, el riesgo ASA (0-IV), el grado de contaminación quirúrgica (herida limpia, limpia-contaminada, contaminada o sucia), la intervención laparoscópica, la adecuación de la preparación prequirúrgica (ducha antiséptica con clorhexidina jabonosa al $2 \%$ y colutorio antiséptico con gluconato de clorhexidina al $0.12 \%$ ), el rasurado del campo quirúrgico, la adecuación de la profilaxis antibiótica (elección del antibiótico, dosis, vía, tiempo de inicio y duración) de acuerdo con la pauta antibiótica en vigor establecida por la comisión de infecciones de nuestro hospital (amoxicilina-ácido clavulánico, $2 \mathrm{~g}$ por vía intravenosa previamente a la inducción anestésica), la presencia de infección de la herida quirúrgica y microorganismo causante.

Para evaluar la adherencia al lavado de manos del personal sanitario se estudiaron la categoría profesional, la técnica de lavado, el antiséptico utilizado y la adecuación de la técnica de lavado a los cinco 
Cirugía y Cirujanos. 2018;86

Tabla 3. Medidas del Plan de Mejora de Calidad y Seguridad Clínica en apendicectomía

1. Campaña de formación a todo el personal sanitario del servicio de cirugía general y del aparato digestivo explicando el plan.

2. Sustitución del lavado higiénico y quirúrgico de manos con clorhexidina por soluciones hidroalcohólicas.

3. Aumento del número de puntos de dispensación de solución hidroalcohólica.

4. Sustitución del rasurado del campo quirúrgico con cuchilla por máquina eléctrica.

5. Antisepsia del campo quirúrgico con clorhexidina alcohólica al 2\% en lugar de povidona yodada.

6. Instalación de dispensadores de solución hidroalcohólica de acción con el codo y relojes temporizadores en quirófanos.

7. Proyección de salvapantallas con recordatorios de las medidas adoptadas en el área de cirugía general y del aparato digestivo.

momentos de higiene de manos recomendados por la Organización Mundial de la Salud (OMS): antes de la atención al paciente, antes de la realización de una técnica aséptica, tras la realización de una técnica aséptica, después de la atención al paciente y después de contactar con el entorno del paciente. La adherencia a la higiene de manos se valoró con observadores anónimos entrenados que realizan habitualmente estudios de observación en centros sanitarios de la Comunidad de Madrid.

Se diseñaron una hoja específica de recogida de datos y una base de datos relacional y normalizada con el programa Microsoft Access ${ }^{\circledR}$ para su registro. Se realizó un estudio descriptivo de la muestra. Las variables cuantitativas se describieron con la media y la desviación estándar (DE) o con la mediana y el rango intercuartílico si no seguían una distribución normal. Se compararon con la prueba t de Student, y si no seguían una distribución normal se usó la prueba $U$ de Mann-Whitney. Las variables cuantitativas con más de dos categorías se compararon con el análisis de la varianza (ANOVA), y si no se cumplían sus condiciones de aplicación se utilizó la prueba no paramétrica de Kruskal-Wallis. Las variables cualitativas se describieron con su distribución de frecuencias y se compararon con la prueba ji al cuadrado de Pearson o con la prueba exacta de Fisher en caso de no cumplir los criterios de aplicación.

Se implantó un Plan de Mejora de Calidad y Seguridad Clínica del paciente quirúrgico durante el año 2011 en el servicio de cirugía general y del aparato digestivo, que se describe en la tabla 3. Se estimó la incidencia acumulada de ISQ en apendicectomías durante dos periodos, antes y después del plan. El año 2011 se excluyó del análisis por haberse implantado en ese año las medidas progresivamente. Se evaluó el efecto de la intervención con la odds ratio (OR) y su intervalo de confianza (IC), ajustando por las diferentes covariables con un modelo de regresión logística backstep por pasos hacia atrás, teniendo en cuenta la confusión y la interacción de las diversas covariables. Para la calibración interna del modelo (bondad de ajuste) se utilizó la prueba de Hosmer-Lemeshow. Los análisis estadístico y epidemiológico se realizaron con los programas SPSS v.22 y Epidat v.4.2. Se consideraron diferencias estadísticamente significativas aquellas con $p<0.05$, y todas las estimaciones se describieron con su IC 95\%.

\section{Resultados}

Se estudiaron 606 pacientes, 267 durante el periodo 2009-2010 (antes del plan) y 339 en el periodo 2012-2013 (después del plan). La edad media fue de 31.7 años (DE: 20). La comorbilidad más frecuente de los pacientes fue la diabetes mellitus $(2.6 \%$ ) y la obesidad (1.8\%). Las características de los pacientes y las principales variables del estudio se muestran en la tabla 4.

Hubo 35 infecciones durante el periodo de seguimiento, lo que supuso una incidencia global de ISQ del $5.78 \%$ (IC 95\%: 4.16-7.95), siendo el $77 \%$ de ellas infecciones de localización superficial. Los microorganismos más frecuentemente implicados fueron Escherichia coli $(37.5 \%)$ y Pseudomonas aeruginosa (25\%). La incidencia de ISQ descendió después de la implantación del plan del $6(n=16)$ al 5.6\% $(n=19)$ (OR: 0.72; IC 95\%: 0.33-1.56; $p=0.839$ ). La estancia media se mantuvo sin cambios significativos, pasando de 4.7 (DE: 7) a 4.6 días (DE: 5) después de la implantación de las medidas preventivas $(p=0.890)$. Los tipos de infección según su profundidad y los microorganismos causantes más frecuentes se describen en la tabla 5.

La adecuación de la profilaxis antibiótica mejoró del 69.2 al $96.2 \%$ después del plan $(p<0.001)$. La 
Tabla 4. Características de los pacientes y principales variables del estudio

\begin{tabular}{|c|c|c|c|}
\hline & $\begin{array}{c}\text { Antes del plan } \\
\mathrm{N}(\%)\end{array}$ & $\begin{array}{c}\text { Después del plan } \\
N(\%)\end{array}$ & $p$ \\
\hline \multicolumn{4}{|l|}{ Sexo } \\
\hline Hombres & $141(52.8)$ & $191(56.3)$ & 0.432 \\
\hline Mujeres & $126(47.2)$ & $148(43.7)$ & 0.432 \\
\hline Total & $267(100)$ & $339(100)$ & \\
\hline $\begin{array}{l}\text { Edad media, } \\
\text { años (DE) }\end{array}$ & $31.8(20)$ & $31.7(19)$ & 0.700 \\
\hline \multicolumn{4}{|l|}{ Comorbilidad } \\
\hline Diabetes mellitus & $7(2.6)$ & $9(2.7)$ & 0.594 \\
\hline Insuficiezncia renal & $4(1.5)$ & $0(0)$ & 0.037 \\
\hline Neoplasia & $3(1.1)$ & $1(0.3)$ & 0.791 \\
\hline EPOC & $2(0.7)$ & $1(0.3)$ & 0.412 \\
\hline Cirrosis hepática & $1(0.4)$ & $0(0)$ & 0.441 \\
\hline Obesidad & $7(2.6)$ & $4(1.2)$ & 0.156 \\
\hline \multicolumn{4}{|l|}{ ASA } \\
\hline I & $180(67.4)$ & $206(60.8)$ & 0.091 \\
\hline$\|$ & $72(27)$ & $112(33)$ & 0.106 \\
\hline III & $14(5.2)$ & $21(6.2)$ & 0.618 \\
\hline IV & $1(0.4)$ & $0(0)$ & 0.441 \\
\hline \multicolumn{4}{|l|}{ NNIS } \\
\hline 0 & $75(28.1)$ & $84(24.8)$ & 0.350 \\
\hline 1 & $126(47.2)$ & $203(59.9)$ & 0.002 \\
\hline 2 & $60(22.5)$ & $44(13)$ & 0.002 \\
\hline 3 & $6(2.2)$ & $8(2.4)$ & 0.920 \\
\hline \multicolumn{4}{|l|}{ Tipo de cirugía } \\
\hline Abierta & $210(78.7)$ & 265 (78.2) & 0.886 \\
\hline Laparoscópica & $46(17.2)$ & $51(15)$ & 0.466 \\
\hline \multicolumn{4}{|l|}{$\begin{array}{l}\text { Duración de la } \\
\text { cirugía }\end{array}$} \\
\hline Media, minutos (DE) & $66.1(34)$ & $57.8(40)$ & 0.045 \\
\hline
\end{tabular}

Tabla 5. Tipos de infección del sitio quirúrgico y microorganismos más frecuentes en apendicectomía

\begin{tabular}{lccc}
\hline & $\begin{array}{c}\text { Antes del plan } \\
\mathbf{N}(\%)\end{array}$ & $\begin{array}{c}\text { Después del plan } \\
\mathbf{N}(\%)\end{array}$ & $\mathbf{p}$ \\
\hline $\begin{array}{l}\text { Tipo de infección } \\
\quad \text { Superficial }\end{array}$ & $11(4.1)$ & $16(4.7)$ & 0.248 \\
$\begin{array}{l}\text { Profunda } \\
\text { De órgano-espacio }\end{array}$ & $3(1.1)$ & $2(0.6)$ & 0.630 \\
Microorganismos & $2(0.7)$ & $1(0.3)$ & 0.238 \\
$\quad$ Escherichia coli & $4(40)$ & $5(37.5)$ & 0.936 \\
$\begin{array}{l}\text { Pseudomona } \\
\text { aeruginosa } \\
\text { Enterobacter cloacae }\end{array}$ & $3(30)$ & $3(21.4)$ & 0.707 \\
\hline
\end{tabular}

causa más frecuente de inadecuación fue el tiempo de inicio, que pasó del 27.9 al $2.8 \%$ tras la implantación de las medidas $(p<0.001)$. Con respecto a la preparación prequirúrgica del paciente, esta aumentó del 3 al 17.5\% ( $p<0.001)$. La principal causa de incumplimiento de la preparación antes del plan fue no administrar la ducha antiséptica al paciente (93.4\%), y tras su implantación, el no usar el antiséptico bucal $(75.6 \%)$.

La adherencia en el Servicio de Cirugía General y del Aparato Digestivo aumentó del 31\% al 40\% ( $p=0,016)$ tras la implantación del plan, destacando el incremento en la adherencia en el $5^{\circ}$ momento de higiene de manos de la OMS (después de contactar con el entorno del paciente), que pasó del $1 \%$ al $36 \%$. En la figura 1 se puede observar la evolución de la adherencia según los momentos, antes y después del Plan de Mejora.

Los factores de riesgo asociados a ISQ fueron diabetes mellitus, preparación prequirúrgica inadecuada, cirugía limpia-contaminada, cirugía sucia, duración de la cirugía mayor del percentil 75, índice de riesgo quirúrgico NNIS 2 y 3 , clasificación ASA > II y uso de drenaje posquirúrgico. La tabla 6 describe el análisis univariante para los diversos factores de riesgo de infección quirúrgica.

En el análisis multivariante se estudiaron los factores de riesgo independientes de ISQ estadísticamente significativos tras el análisis univariante y aquellos con $p$ $\leq 0.2$ que se consideraron de interés por su trascendencia clínica y pronóstica (insuficiencia renal, neoplasia, rasurado y cirugía de urgencia). Tras el análisis multivariante mostraron significación estadística la clasificación ASA > II (OR: 5.66; IC 95\%: 2.25-14.26), el uso de drenaje posquirúrgico (OR: 6.37; IC 95\%: 2.6615.25) y la cirugía limpia-contaminada (OR: 0.20; IC 95\%: 0.58-0.70).

\section{Discusión}

La existencia de tasas bajas de ISQ es proporcional a una adecuada práctica quirúrgica y representa un buen indicador de la mejora de la calidad y la seguridad de la asistencia sanitaria prestada en cada centro ${ }^{13,14}$. Las acciones dirigidas a la prevención de las infecciones son siempre una medida costo-efectiva, por lo que, en el actual contexto de recursos limitados, también representa un valor añadido para la sostenibilidad de los sistemas sanitarios ${ }^{15,16}$.

La evaluación de la incidencia de infección requiere la vigilancia de los procedimientos quirúrgicos que respondan a cirugías frecuentes, asociados a un riesgo relativamente alto de ISQ y que tengan consecuencias graves para el paciente en caso de que se produzca una infección. Cualquier tipo de cirugía supone un riesgo de infección, y en la apendicectomía, que es 


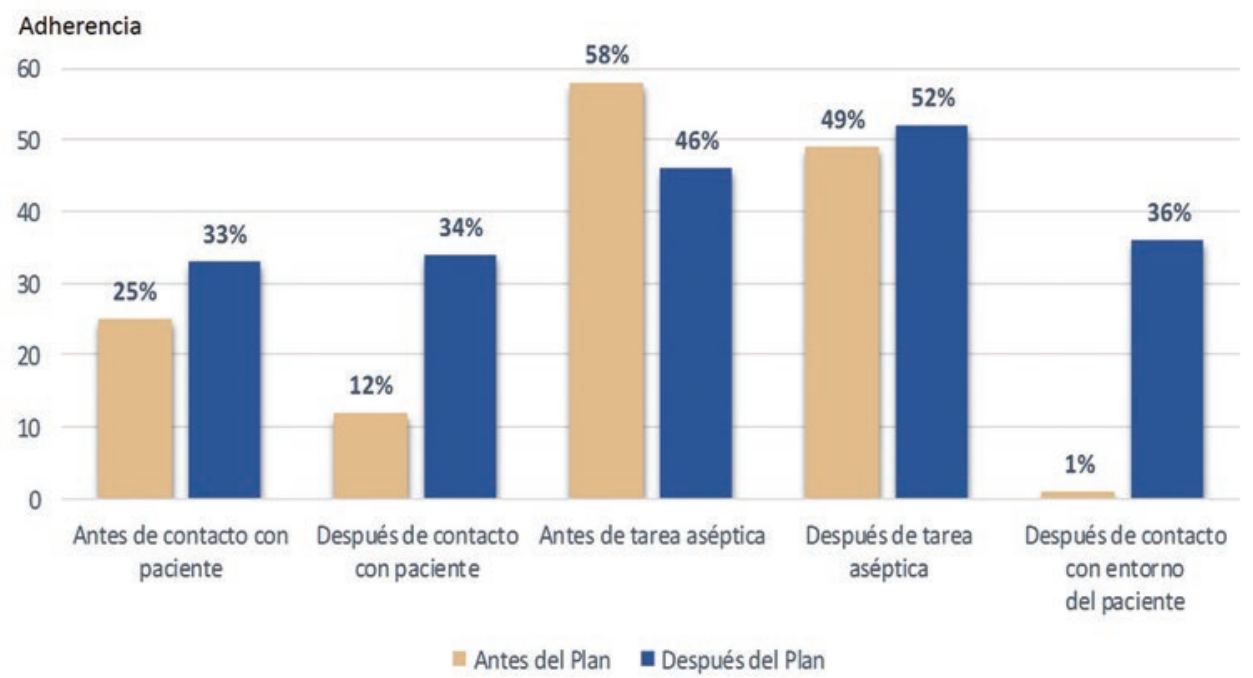

Figura 1. Evolución de la adherencia a la higiene de manos, según los momentos recomendados por la Organización Mundial de la Salud, antes y después del Plan de Mejora de Calidad y Seguridad Clínica.

uno de los procedimientos quirúrgicos más realizados, se deben extremar los cuidados asistenciales ${ }^{17}$.

La incidencia global de ISQ durante el periodo de estudio fue del $5.8 \%$, y tras la aplicación de las medidas preventivas disminuyó del 6 al 5.6\%; una cifra tan baja en nuestro medio no se encontró en la literatura revisada, en la que la mayoría de las series no descienden de un $8 \%$ de incidencia ${ }^{18-20}$. Al igual que en otros estudios, los microorganismos aislados con más frecuencia en pacientes con infección quirúrgica tras una apendicectomía fueron bacilos gramnegativos, en concreto $E$. coli y $P$. aeruginosa ${ }^{21,22}$.

Algunos trabajos evalúan la incidencia al alta de los pacientes sin hacer un seguimiento del periodo completo de riesgo ${ }^{23}$, lo que dificulta la comparación de las cifras de incidencia de infección si solo se sigue a los pacientes durante el ingreso hospitalario y no durante los 30 días tras la cirugía. Hasta la mitad de las infecciones en apendicectomías suceden tras el alta hospitalaria ${ }^{18}$. En nuestro caso, el seguimiento se ha realizado durante los 30 días del posoperatorio para no infravalorar la incidencia de ISQ.

Respecto al Plan de Mejora de Calidad y Seguridad Clínica implantado, una aplicación continuada de medidas preventivas como las utilizadas en nuestro hospital puede prevenir del 33 al $60 \%$ de estas infecciones, según la literatura consultada ${ }^{10,12,24,25}$.

La ISQ es una de las principales causas de infección nosocomial, y por tanto son esenciales las medidas preventivas para disminuir su incidencia, coste y mortalidad. Los pacientes con infección tras una apendicectomía tienen un coste medio para el sistema sanitario tres veces superior a los no infectados ${ }^{26}$.
Aunque la aplicación del plan redujo la incidencia de ISQ en nuestro centro, la estancia media se mantuvo sin cambios apreciables. Pérez Blanco, et al. ${ }^{27}$, en un estudio realizado en nuestro entorno, obtuvieron una reducción de la estancia media y de la infección quirúrgica del 27.5 al $16.9 \%(p>0.05)$ tras evaluar un protocolo de medidas preventivas en pacientes de cirugía colorrectal. En este sentido, Izquierdo Blasco, et al. ${ }^{28}$, en un estudio cuasi-experimental en pacientes pediátricos de cirugía cardiaca en un hospital español, observaron una disminución de ISQ del $10.9 \%$ al $1.9 \%(p<0.05)$. Otras revisiones internacionales lograron resultados favorables similares a los descritos ${ }^{13,29-32}$.

Uno de los mayores logros tras la implantación de nuestro plan fue la mejoría del cumplimiento del protocolo de profilaxis antibiótica, que pasó del 69.2 al $96.2 \%$ ( $p<0.001$ ), con una adecuación global del $81.9 \%$, considerando una administración correcta si se cumplían todos los criterios (elección del antibiótico, dosis, tiempo de inicio, vía de administración y duración). Estas cifras son superiores a las de otros trabajos nacionales e internacionales revisados ${ }^{7,22,33-35}$. La causa de inadecuación más frecuente fue no administrar la profilaxis en el momento previo a la cirugía definido en el protocolo. Varias series de la literatura coinciden con este hallazgo $0^{7,22,34,35}$.

La explicación de no administrar la profilaxis en el momento previo a la cirugía se debe a que las apendicitis son diagnosticadas en los servicios de urgencia y esto hace que pueda existir cierto retraso entre la valoración del paciente, la administración de la profilaxis y la cirugía. La profilaxis fue administrada por personal 
Tabla 6. Análisis univariante para los factores de riesgo de infección del sitio quirúrgico en apendicectomía

\begin{tabular}{|c|c|c|c|c|c|c|}
\hline \multirow[t]{2}{*}{ Factores de riesgo } & \multirow{2}{*}{$\begin{array}{c}\text { ISQ } \\
\mathrm{N}(\%)\end{array}$} & \multirow{2}{*}{$\begin{array}{c}\text { No ISQ } \\
\text { N (\%) }\end{array}$} & \multirow{2}{*}{$\begin{array}{c}\text { Total } \\
\mathrm{N}(\%)\end{array}$} & \multirow[t]{2}{*}{ OR } & \multirow[t]{2}{*}{ IC $95 \%$} & \multirow[t]{2}{*}{$\mathrm{p}$} \\
\hline & & & & & & \\
\hline Antes del plan & $16(6)$ & $251(94)$ & $267(44.1)$ & 1.07 & $0.54-2.13$ & 0.839 \\
\hline Después del plan & $19(5.6)$ & $320(94.4)$ & $339(55.9)$ & 0.93 & $0.47-1.85$ & 0.839 \\
\hline Sexo masculino & $21(60)$ & $311(54.5)$ & $332(54.8)$ & 1.24 & $0.62-2.46$ & 0.538 \\
\hline Sexo femenino & $14(40)$ & $260(45.5)$ & $274(45.2)$ & 0.81 & $0.41-1.60$ & 0.538 \\
\hline Diabetes mellitus & $3(8.6)$ & $13(2.3)$ & $16(2.6)$ & 4.02 & $1.09-14.84$ & 0.049 \\
\hline Insuficiencia renal & $1(2.9)$ & $3(0.5)$ & $4(0.7)$ & 5.57 & $0.56-54.96$ & 0.212 \\
\hline Neoplasia & $1(2.9)$ & $3(0.5)$ & $4(0.7)$ & 5.57 & $0.56-54.96$ & 0.212 \\
\hline EPOC & $0(0)$ & $3(0.5)$ & $3(0.5)$ & - & - & - \\
\hline Cirrosis hepática & $0(0)$ & $1(0.2)$ & $1(0.2)$ & - & - & - \\
\hline Neutropenia & $0(0)$ & $0(0)$ & $0(0)$ & - & - & - \\
\hline Obesidad & $1(2.9)$ & $10(1.8)$ & $11(1.8)$ & 2.33 & $0.41-13.31$ & 0.483 \\
\hline Desnutrición & $0(0)$ & $0(0)$ & $0(0)$ & - & - & - \\
\hline Rasurado & $10(28.6)$ & $118(20.7)$ & $128(21.1)$ & 1.58 & $0.75-3.32$ & 0.229 \\
\hline Profilaxis quirúrgica inadecuada & $1(2.9)$ & $12(2.1)$ & $13(2.1)$ & 1.79 & $0.22-14.49$ & 0.457 \\
\hline $\begin{array}{l}\text { Preparación quirúrgica } \\
\text { inadecuada }\end{array}$ & $27(77.1)$ & $511(89.5)$ & $538(88.8)$ & 0.39 & $0.17-0.90$ & 0.030 \\
\hline Cirugía de urgencia & $19(54.3)$ & $265(46.4)$ & $284(46.9)$ & 1.37 & $0.69-2.72$ & 0.232 \\
\hline Laparoscopia & $4(11.4)$ & $93(16.3)$ & $97(16)$ & 0.66 & $0.23-1.92$ & 0.635 \\
\hline Cirugía limpia-contaminada & $3(8.6)$ & $205(35.9)$ & $208(34.3)$ & 0.23 & $0.08-0.65$ & 0.001 \\
\hline Cirugía contaminada & $10(28.6)$ & $176(30.8)$ & $186(30.7)$ & 0.90 & $0.42-1.91$ & 0.831 \\
\hline Cirugía sucia & $21(60)$ & $188(32.9)$ & $209(34.5)$ & 3.06 & $1.52-6.14$ & 0.001 \\
\hline Duración de la cirugía>percentil 75 & $15(42.9)$ & $133(23.3)$ & $148(24.4)$ & 2.47 & $1.23-4.96$ & 0.011 \\
\hline$A S A>2$ & $9(25.7)$ & $27(4.7)$ & $36(5.9)$ & 6.97 & $2.98-16.33$ & 0.000 \\
\hline NNIS 2 & $12(34.3)$ & $92(16.1)$ & $104(17.2)$ & 2.72 & $1.31-5.65$ & 0.009 \\
\hline NNIS 3 & $5(14.3)$ & $9(1.6)$ & $14(2.3)$ & 10.41 & $3.28-32.98$ & 0.001 \\
\hline Drenaje posquirúrgico & $10(28.6)$ & $28(4.9)$ & $38(6.3)$ & 7.76 & $3.40-17.72$ & 0.000 \\
\hline Transfusión sanguínea & $0(0)$ & $2(0.4)$ & $2(0.3)$ & - & - & - \\
\hline
\end{tabular}

ASA: American Society of Anesthesiologists; EPOC: enfermedad pulmonar obstructiva crónica; IC 95\%: intervalo de confianza del 95\%; ISQ: infección de sitio quirúrgico; NNIS: National Nosocomial Infections Surveillance; OR: odds ratio.

de enfermería de quirófano, de acuerdo con el protocolo del hospital y supervisada por los anestesiólogos. Ninguno de ellos sabía que serían evaluados, por lo que se controló el efecto Hawthorne ${ }^{36}$.

La preparación quirúrgica constituye un elemento fundamental en la prevención de la infección de la herida. La piel del paciente es un reservorio de microorganismos saprófitos residentes transitorios de fácil eliminación con el lavado con agua y jabón.
También hay una flora residente que necesita antisepsia para su eliminación ${ }^{37-39}$. En el estudio se consideró como preparación incorrecta la falta de aplicación del antiséptico bucal o del lavado corporal.

En nuestra serie hubo un $11.1 \%$ de cumplimiento global de la preparación prequirúrgica, que mejoró del 3 al $17.5 \%(p<0.001)$ después del plan. La principal causa de inadecuación antes del plan fue la no preparación del paciente, y tras el plan fue la no 
administración del antiséptico bucal. Si comparamos con otros estudios, la preparación quirúrgica correcta de nuestros pacientes es menor ${ }^{25,40,41}$, lo que se explica, como sucedía con la profilaxis antibiótica, por el hecho de que la apendicectomía es una técnica quirúrgica urgente y el paciente pasa de la sala de urgencias al quirófano directamente y no da tiempo a la aplicación de las medidas preventivas.

El Plan de Mejora de Calidad y Seguridad Clínica incluyó también medidas para la promoción de la higiene de manos en el personal sanitario. La importancia del lavado de manos en la transmisión de las infecciones nosocomiales está muy bien documentada. La higiene de manos es reconocida como la medida más importante para la prevención de las infecciones asociadas a la asistencia sanitaria ${ }^{42,43}$. Con una adecuada higiene de manos se pueden prevenir más del $50 \%$ de las infecciones en el ámbito sanitario. Esta práctica no está suficientemente reconocida por los profesionales, por lo que existe una baja adherencia a los cinco momentos de higiene de manos recomendados por la OMS ${ }^{42,44}$.

Las soluciones hidroalcohólicas permiten una desinfección de las manos de modo sencillo, barato y efectivo, y son parte de las medidas preventivas de nuestro plan. Después del año 2011 se objetivó una mayor adherencia al lavado de manos en nuestro centro, pasando del 37 al 42\% ( $p<0.05)$; concretamente, en el servicio de cirugía general y del aparato digestivo pasó del 31 al 40\% ( $p<0.05)$, cifras similares a las reportadas en el estudio de Sánchez-Payá, et al. ${ }^{45}$ en otro hospital universitario español.

Aunque el diseño de este estudio es de alta evidencia científica, una posible limitación sería no haber podido captar algunas infecciones quirúrgicas del periodo posoperatorio, ocurridas extrahospitalariamente, que no hubieran sido registradas en la historia clínica. Sin embargo, estas son muy infrecuentes y pensamos que nuestros resultados no se han visto afectados por este hecho.

Para evitar sesgos por pérdidas en el seguimiento, al realizar el cálculo del tamaño muestral se estimó un porcentaje de posibles pérdidas durante el periodo evaluado. Nuestro centro dispone de historia clínica electrónica y los pacientes son seguidos en atención primaria con la aplicación informática Horus ${ }^{\circledR}$ tras el alta hospitalaria, por lo que los sesgos de selección e información también han podido ser controlados.

Como consecuencia del estudio se tomaron diversas medidas en nuestro hospital, entre ellas comunicar los resultados a los médicos responsables para incrementar el cumplimiento de estas recomendaciones, ya que hay evidencia de que una evaluación de los procedimientos basada en el conocimiento de los resultados (feedback) por los cirujanos puede disminuir de forma significativa las tasas de infección ${ }^{27}$.

\section{Conclusiones}

Un paquete de medidas preventivas es un conjunto de medidas y prácticas basadas en la evidencia que, cuando se aplican de manera agrupada, mejoran la atención sanitaria. Las infecciones quirúrgicas representan un importante problema para la seguridad del paciente en los sistemas de salud, limitan los beneficios de la cirugía y suponen un riesgo adicional en términos de morbimortalidad y aumento de costes.

En nuestro hospital, la incidencia de ISQ en apendicectomías fue baja, pero siempre es susceptible de mejora. A pesar de que la reducción en la incidencia de infección no tuvo significación estadística tras las medidas adoptadas, en poco tiempo hemos logrado importantes progresos en campos como la adecuación de la profilaxis antibiótica, la adherencia a la higiene de manos y la preparación prequirúrgica del paciente, que creemos que pueden servir de referencia a otros centros sanitarios de similares características. Consideramos esencial la perseverancia y el cumplimiento de las medidas preventivas iniciadas, así como su prolongación y evaluación de forma continuada, para la mejora futura de este Plan de Mejora de Calidad y Seguridad Clínica y su implantación en la totalidad de las cirugías subsidiarias de ello.

\section{Agradecimientos}

Los autores agradecen al Fondo de Investigación Sanitaria (FIS, proyecto n.. PI11/01272) y a los fondos europeos de desarrollo regional la financiación del trabajo.

\section{Conflicto de intereses}

Los autores declaran no tener ningún conflicto de intereses.

\section{Bibliografía}

1. Rodríguez MA, Begerano N, Pérez N, Pedroso MV, Regla C. The infections associated with the health care. Invest Medicoquir. 2014;6:147-57. 
2. Yokoe DS, Classen D. Improving patient safety through infection control: a new healthcare imperative. Infect Control Hosp Epidemiol. 2008;29(Suppl 1):S3-S11.

3. Dellinger EO, Gross PA, Barrett TL, Krause PJ, Martone WJ, McGowan JE, et al. Quality standard for antimicrobial prophylaxis in surgical procedures. The Infectious Diseases Society of America. Infect Control Hosp Epidemiol. 1994;15:182-8.

4. Ángeles-Garay U, Morales-Márquez LI, Sandoval-Balanzarios MA, Velázquez-García JA, Maldonado-Torres L, Méndez-Cano AF. Risk factors related to surgical site infection in elective surgery. Cir Cir. 2014;82:48-62.

5. Sociedad Española de Medicina Preventiva, Salud Pública e Higiene. 2017. EPINE. Estudio de Prevalencia de las Infecciones Nosocomiales en España. (Consultado el 14-2-2018.) Disponible en: http://hws.vhebron.net/epine/

6. Del Moral Luque JA, Alonso García M, Gil Yonte P, Fernández Cebrián JM Durán Poveda M, Rodríguez Caravaca G. Incidence of surgical site infection in colon surgery and antibiotic prophylaxis adequacy: prospective cohort study. An Sist Sanit Navar. 2017;40:371-7.

7. Díaz-Agero Pérez C, Robustillo Rodela A, Pita López MJ, López Fresneña N, Monge Jodrá V. Surgical wound infection rates in Spain: data summary, January 1997 through June 2012. Am J Infect Control. 2014;42:521-4.

8. García Sabrido JL. Apendicectomía laparoscópica frente a apendicectomía abierta: relatividad de resultados y eficacia. Cir Esp. 2000;67:221-2.

9. Kirkland KB, Bridges JP, Trivette SL, Wilkinson WE, Sexton DJ. The impact of surgical site infections in the 1990s: attributable mortality, excess length of hospitalization and extra costs. Infect Control Hosp Epidemiol. 1999;20:725-30.

10. Anderson DJ, Podgorny K, Berríos-Torres SI, Bratzler DW, Dellinger EP, Greene $L$, et al. Strategies to prevent surgical site infections in acute care hospitals: 2014 update. Infect Control Hosp Epidemiol. 2014;35(Suppl 2):S66-88.

11. Leaper D, McBain AJ, Kramer A, Assadian O, Sánchez JL, Lumio J, et al. Healthcare associated infection: novel strategies and antimicrobial implants to prevent surgical site infection. Ann R Coll Surg Engl. 2010;92:453-8.

12. Fariñas-Álvarez C, Teira-Cobo R, Rodríguez-Cundín P. Infección asociada a cuidados sanitarios (infección nosocomial). Medicine. 2010; 10:3293-300.

13. Wick EC, Hobson DB, Bennett JL, Demski R, Maragakis L, Gearhart SL, et al. Implementation of a surgical comprehensive unit-based safety program to reduce surgical site infections. J Am Coll Surg. 2012;215:193-200.

14. Sparkes D, Rylah B. The World Health Organization surgical safety checklist. Br J Hosp Med (Lond). 2010;71:276-80.

15. Rocha-Almazán M, Sánchez-Aguilar M, Belmares-Taboada J, Esmer-Sánchez D, Tapia-Pérez JH, Gordillo-Moscoso A. Infección del sitio operatorio en cirugía abdominal no traumática. Cir Cir. 2008;76:127-31.

16. Cárdenas-Salomón CM, Cervantes-Castro J, Jean-Silver ER, Toledo-Valdovinos SA, Murillo-Zolezzi A, Posada-Torres JA. Hospitalization costs of open vs. laparoscopic appendectomy: 5-year experience. Cir Cir. 2011;79:534-9.

17. Aguiló J, Peiró S, Muñoz C, García del Caño J, Garay M, Viciano V, et al. Efectos adversos en la cirugía de la apendicitis aguda. Cir Esp. 2005;78:312-7.

18. Aranda-Narváez JM, Prieto-Puga Arjona, T García-Albiach B, Montiel-Casado MC, González-Sánchez AJ, Sánchez-Pérez B, et al. Infección de sitio quirúrgico tras apendicectomía urgente: tasa global y tipo según la vía de abordaje (abierta/laparoscópica). Enferm Infecc Microbiol Clin. 2014;32:76-81.

19. Íñigo JJ. Infección de sitio quirúrgico en un servicio de cirugía general. Análisis de cinco años y valoración del índice National Nosocomial Infection Surveillance (NNIS). Cir Esp. 2006;79:224-30.

20. Monge-Jodrá V, Robustillo-Rodela A, Martín-Martínez F, López-Fresneña N. Quality Control Indicator Working Group. Standardized infection ratios for three general surgery procedures: a comparison between Spanish hospitals and U.S. centers participating in the National Nosocomial Infections Surveillance System. Infect Control Hosp Epidemiol. 2003;24:744-8.

21. Sutter VI. Frequency of occurrence and antimicrobial susceptibility of bacterial isolates from intestinal and female genital tracts. Rev Infect Dis. 1983;5(Suppl 1):S84-9.

22. Sánchez-Santana T, Del-Moral-Luque JA, Gil-Yonte P, Bañuelos-Andrío L, Durán-Poveda M, Rodríguez-Caravaca G. Efecto de la adecuación a protocolo de la profilaxis antibiótica en la incidencia de infección quirúrgica en apendicectomías. Estudio de cohortes prospectivo. Cir Cir. 2017;85:208-13.

23. Jodrá VM, Díaz-Agero Pérez C, Sáinz de los Terreros Soler L, Saa Requejo CM, Dacosta Ballesteros D. Quality Control Indicator Working
Group. Results of the Spanish national nosocomial infection surveillance network (VICONOS) for surgery patients from January 1997 through December 2003. Am J Infect Control. 2006;34:134-41.

24. Ruiz Tovar J, Badía JM. Medidas de prevención de la infección del sitio quirúrgico en cirugía abdominal. Revisión crítica de la evidencia. Cir Esp. 2014;92:223-31.

25. Díaz-Agero Pérez C, Pita-López MJ, Robustillo-Rodela A, Figuerola-Tejerina A, Monge-Jodrá V. Evaluación de la infección de herida quirúrgica en 14 hospitales de la Comunidad de Madrid: estudio de incidencia. Enferm Infecc Microbiol Clin. 2011;29:257-62.

26. Ríos J, Murillo C, Carrasco G, Humet C. Incremento de costes atribuible a la infección quirúrgica de la apendicectomía y colectomía. Gac Sanit. 2003;17:218-25.

27. Pérez-Blanco V, García-Olmo D, Maseda-Garrido E, Nájera-Santos MC, García-Caballero J. Evaluación de un paquete de medidas para la prevención de la infección de localización quirúrgica en cirugía colorrectal. Cir Esp. 2015;93:222-8.

28. Izquierdo-Blasco J, Campins-Martí M, Soler-Palacín P, Balcells J, Abella R, Gran F, et al. Impact of the implementation of an interdisciplinary infection control program to prevent surgical wound infection in pediatric heart surgery. Eur J Pediatr. 2015;174:957-63.

29. Waits SA, Fritze D, Banerjee M, Zhang W, Kubus J, Englesbe MJ, et al. Developing an argument for bundled interventions to reduce surgical site infection in colorectal surgery. Surgery. 2014;155:602-6.

30. Lutfiyya W, Parsons D, Breen J. A colorectal "care bundle" to reduce surgical site infections in colorectal surgeries: a single-center experience. Perm J. 2012;16:10-6.

31. Keenan JE, Speicher PJ, Thacker JK, Walter M, Kuchibhatla M, Mantyh CR. The preventive surgical site infection bundle in colorectal surgery: an effective approach to surgical site infection reduction and health care cost savings. JAMA Surg. 2014;149:1045-52.

32. Crolla RM, van der Laan $L$, Veen EJ, Hendriks $Y$, van Schendel C, Kluytmans J. Reduction of surgical site infections after implementation of a bundle of care. PLoS One. 2012;7:1-6.

33. Forbes SS, Stephen WJ, Harper WL, Loeb M, Smith R, Christoffersen EP, et al. Implementation of evidence-based practices for surgical site infection prophylaxis: results of a pre- and postintervention study. J Am Coll Surg. 2008;207:336-41.

34. Sviestina I, Mozgis D. Evaluation of the antibiotic use for surgical prophylaxis in paediatric acute appendicitis. J Young Pharm. 2015;7:7-11.

35. Kasatpibal N, Nørgaard M, Sørensen HT, Schønheyder HC, Jamulitrat S, Chongsuvivatwong V. Risk of surgical site infection and efficacy of antibiotic prophylaxis: a cohort study of appendectomy patients in Thailand. BMC Infect Dis. 2006;6:111.

36. McCarney R, Warner J, lliffe S, van Haselen R, Griffin M, Fisher P. The Hawthorne effect: a randomised, controlled trial. BMC Med Res Methodol. $2007 ; 7: 30$.

37. Darouiche RO, Wall MJ, Itani KM, Otterson MF, Webb AL, Carrick MM, et al. Chlorhexidine-alcohol versus povidone-iodine for surgical-site antisepsis. N Engl J Med. 2010;362:18-26.

38. Tanner J, Khan D. Surgical site infection, preoperative body washing and hair removal. J Perioper Pract. 2008;18:237-42.

39. Chauveaux D. Preventing surgical-site infections: measures other than antibiotics. Orthop Traumatol Surg Res. 2015;101(Suppl 1):S77-83.

40. Rodríguez-Caravaca G, Gil-Yonte P, Del-Moral-Luque JA, Covelé-Lucas W, Fernández-Cebrián JM, Durán-Poveda M. Rates of surgical site infection in cholecystectomy: comparison between a University Teaching Hospital, Madrid Region, Spain and USA rates. Rev Invest Clin. 2017;69:336-43.

41. Hijas-Gómez Al, Egea-Gámez RM, Martínez-Martín J, González-Díaz R, Losada-Viñas JI, Rodríguez-Caravaca G. Surgical wound infection rates and risk factors in spinal fusion in a University Teaching Hospital in Madrid, Spain. Spine. 2017; 42:748-54.

42. Organización Mundial de la Salud. Prevención de las infecciones nosocomiales. Guía Práctica. 2. a ed. Malta: OMS; 2002. 65 p.

43. Organización Mundial de la Salud. Guía de la OMS sobre higiene de manos en la atención de la salud: resumen. Primer desafío global de seguridad del Paciente. Una atención limpia es una atención segura. Geneva: OMS; 2009. 80 p.

44. Pittet D, Hugonnet S, Harbarth S, Mourouga P, Sauvan V, Touveneau S, et al. Effectiveness of a hospital-wide programme to improve compliance with hand hygiene. Infection Control Programme. Lancet. 2000; 356:1307-12.

45. Sánchez-Payá J, Fuster-Pérez M, García-González C, Gracia-Rodríguez RM, García-Shimizu P, San Juan-Quiles R, et al. Evaluación de un programa de actualización de las recomendaciones sobre la higiene de manos. An Sist Sanit Navar. 2007;30:343-52. 